\title{
El principio de no-contradicción en la argumentación escéptica: implicaciones \\ y consecuencias
}

\section{The Principle of Non-Contradiction in the Skeptic Argumentation: Implications and Consequences}

\author{
Ignacio PAJÓN LeYRA \\ Universidad Complutense de Madrid
}

Recibido: 23-05-2011

Aceptado: 23-06-2011

\section{Resumen}

El empleo por parte del escepticismo pirrónico de un esquema argumentativo basado en la noción de contradicción nos induce a pensar que dicha noción juega un papel importante entre los puntos de partida básicos de su filosofía. Por ello, se muestra como imprescindible emprender un análisis pormenorizado de las implicaciones que conlleva la presencia de esta noción en el argumento escéptico clásico para determinar la presencia de implicaciones derivadas del principio de no-contradicción y su compatibilidad con la posición filosófica propia de esta corriente helenística.

Palabras clave: Aristóteles, contradicción, escepticismo, Sexto Empírico.

\begin{abstract}
The use, by the pyrronian scepticism, of an argumentative pattern based on the notion of contradicition leads us to think that said notion plays an important role among the basic starting points of its philosophy. Therefore, it appears as essential to start a detailed analysis of the implications of this notion inside the classic sceptical argument, in order to determinate the presence of implications derived from
\end{abstract}


the principle of non-contradiction, and their compatibility with the philosophical position that corresponds to this hellenistic school.

Keywords: Aristotle, contradiction, skepticism, Sextus Empiricus.

En el funcionamiento interno de la filosofía escéptica, la contradicción es una de las nociones que desempeña un papel más importante. La manera de proceder escéptica en sus argumentaciones se basa en el mecanismo de poner "en oposición" apariencias con apariencias, pensamientos con pensamientos, o uno de ellos con el otro, tal y como Sexto Empírico expone en el capítulo primero, parágrafo 31 de los Esbozos pirrónicos. Así, al producirse el equilibrio entre ambos polos de la oposición, la elección racional queda imposibilitada, con lo que el argumento escéptico alcanza su objetivo de conducir a la suspensión del juicio. La labor del escéptico es encontrar la apariencia o pensamiento adecuado para oponer a cualquier apariencia o pensamiento, y situarlos en directa oposición. Si alguno quedase sin oposición, nada impediría afirmar la realidad de lo que esa apariencia o pensamiento muestra, con lo que se truncaría la suspensión del juicio y no se llegaría a alcanzar la ataraxía. Sin oposición, pues, no hay escepticismo.

Esta estructura argumetativa nos induce a pensar que la noción de contradicción presente en la filosofía escéptica conlleva la vigencia de alguna de las formulaciones del principio de no-contradicción. Sin embargo, la asunción de un principio lógico-ontológico como este supera claramente los límites del ámbito de lo manifiesto, que la corriente pirrónica se ha marcado como único espacio de su actividad filosófica. De este modo, un análisis de las implicaciones de la presencia del principio de no-contradicción en los argumentos escépticos se nos muestra como irrenunciable a la hora de determinar la coherencia de la posición pirrónica en pleno.

Para llevar a cabo dicho análisis consideramos que será necesario contemplar el ejemplo de argumentación escéptica que suponen los Esbozos pirrónicos a la luz del tratamiento del principio de no-contradicción ${ }^{1}$ que Aristóteles lleva a cabo en la Metafísica, en especial en el fragmento que abarca desde 1005 b 35 hasta 1009 a 5.

Aristóteles describe el PNC como un principio "acerca del cual no es posible caer en error, sino que siempre se hace necesariamente lo contrario, o sea, estar en la verdad"2.

Se trata de un principio indemostrable, ya que cualquier demostración que pretendiera hacerse de él tendría que deducirlo a partir de otro principio dotado de mayor certeza. Y no es posible mayor grado de certeza que el que posee un principio cuya verdad es necesaria.

\footnotetext{
1 En adelante, PNC.

2 1061 b 34-5.
} 
Sobre el PNC no es posible equivocarse en lo que se refiere a su verdad; no es posible cometer un verdadero error. Podríamos preguntarnos, entonces, qué sentido tiene dedicarle a ese principio autoevidente tanto espacio en la Metafísica ${ }^{3}$. La respuesta probablemente se encuentra en la misteriosa figura dialéctica a la que el Estagirita se enfrenta a partir de 1005 b 35: los que pretenden negar el PNC.

Puede pensarse que Aristóteles habla de un negador hipotético de este principio; que argumenta contra posiciones teóricas con independencia de que hayan sido asumidas de hecho por alguien o no ${ }^{4}$. Sin embargo, tratándose de un principio autoevidente, sería más fácil entender la insistencia del Estagirita si no se interpretase como un mero ejercicio teórico orientado a cubrir un posible ámbito de argumentación poco probable, sino de la refutación efectiva de una argumentación real y de algún modo vigente en la controversia filosófica del momento ${ }^{5}$.

Por ese motivo, los intentos de identificación del interlocutor de Aristóteles en este pasaje han sido múltiples y muy variados. Y entre los muchos candidatos siempre ha tenido un lugar de honor el escepticismo.

En efecto, el método de contraposición escéptico parece encajar bastante bien, en general, con el esquema básico que Aristóteles atribuye al negador del principio. Para Aristóteles, esta negación consistiría en la afirmación simultánea de las proposiciones opuestas 6 . Es decir: quien dice que algo es y no es esto o aquello, estará negando dicho principio ${ }^{7}$. El escéptico, por su parte, contrapone afirmaciones opuestas en su búsqueda de la epoché, de modo que señalará que algo aparece unas

\footnotetext{
3 En concreto de 1005 b 35 a 1009 a 5 y de 1061 b 34 a 1063 b 34.

${ }^{4}$ Uno de los motivos que han llevado a considerar esta posibilidad es que el propio Aristóteles nunca llega a identificar claramente a su adversario argumentativo. Esto, sin embargo, no parece indicio suficiente para sustentar la tesis en cuestión, en especial si se tiene en cuenta que no es el único pasaje en que Aristóteles cita una posición filosófica de manera anónima. Sobre este particular, Colli señala que: "Aristóteles no se guía por el interés histórico de testimoniar y transmitir las posiciones de los filósofos precedentes, sino por un interés teórico ante las posiciones asumidas por el pensamiento anterior a él; de ahi que las citas de Aristóteles sean a menudo polémicas y anónimas. El anonimato muestra que Aristóteles no tiene un interés histórico sino teórico, ya que no siempre es posible explicarlo como una referencia a opiniones cuya atribución no era difícil para los contemporáneos." (Colli, G. Zenone di Elea. Lezioni 1964-1965. Adelphi Edizioni, Milano, 1998. Traducción española de Miguel Morey, Zenón de Elea. Editorial Sexto Piso, Madrid, 2006: 71). Sin embargo, esto supondría que aunque no hay en Aristóteles interés histórico, sino sólo teórico por las posiciones filosóficas precedentes, en general se trata de un interés teórico por las posiciones filosóficas históricamente defendidas por el pensamiento anterior.

5 Además, también la propia redacción del principio del capítulo cuarto del libro IV de la Metafísica nos da a entender que no se trata de una posición teóricamente sostenible, sino sostenida de facto por alguien. ("Hay, como decíamos, algunos que afirman que lo mismo puede ser y no ser y que es posi-

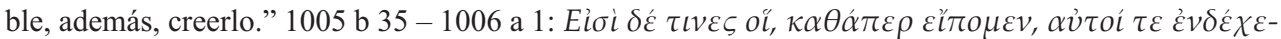

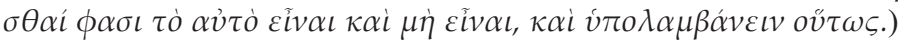

61062 a 5.

7 vid. 1065 a $15-20$.
} 
veces como siendo esto, otras como siendo aquello. Así, puede parecer que es en el escepticismo en donde se encuentra la posición más próxima a la que Aristóteles describe. Ambas emplean simultáneamente proposiciones opuestas como base para su argumentación. Por supuesto, no deja de estar presente el insalvable problema que supone la incongruencia temporal de una identificación del oponente de Aristóteles con el escepticismo.

Pero ese grave problema aún pueden tratar de salvarlo los partidarios de esta interpretación afirmando que la posición filosófica que Aristóteles describe es escepticismo, aun cuando no se trate de escepticismo pirrónico. Podría atribuirse, pues, a una anticipación del escepticismo posterior por parte de alguno de sus antecedentes históricos.

Pero dejando por el momento de lado la conveniencia o no de los personajes concretos a los que podría adjudicarse la defensa de esta posición, se hace necesario considerar si el problema de la inconsistencia temporal es el único que impide la plena identificación del negador del PNC como escéptico.

Teniendo en cuenta sólo el contenido temático de ambas posiciones encontramos que el interlocutor del pasaje aristotélico afirma proposiciones opuestas, mientras que el escéptico contrapone afirmaciones opuestas. La aparente similitud, basada en el uso simultaneo de proposiciones de signo contrario y en la propia noción de oposición, se revela, en un análisis más profundo, como un mero espejismo.

Aunque la noción de oposición esté presente en ambas argumentaciones, el uso que se hace de ella es diferente. Mientras que el negador del PNC, como decimos, afirma ambos polos de la oposición -y es necesario que los afirme para que sea tal negador-, el escéptico emplea afirmaciones previas a su propia argumentación. No es él quien afirma, sino que emplea en la mayoría de los casos afirmaciones ajenas, y excepcionalmente incluso afirmaciones sólo posibles8.

Por lo tanto, el empleo del método escéptico de contraposición no implicaría de inmediato la ruptura del PNC. Se trataría, más bien, de un enfrentamiento entre argumentaciones sin compromiso asertivo por parte del escéptico con ninguna de ellas -y desde luego menos aún con ambas a un tiempo-. Es más, desde nuestro punto de vista, el PNC, ha de seguir vigente para que el argumento escéptico tenga sentido. El objetivo de la contraposición de opuestos es la isosthéneia, la equipolencia, el equilibrio de fuerzas entre ambos polos de la contraposición. Ambos argumentos han de tener la misma capacidad de convicción, han de ser igual de fuertes en lo que a su fuerza persuasiva se refiere, de tal modo que la elección racional entre ellos sea imposible. Los argumentos así contrapuestos se anularán entre ellos sumiendo al filósofo en la epochế. Pero sólo se anularán si la elección entre ellos es imposible, y al tiempo son inasumibles de manera simultánea. E incluso habría un

${ }^{8} H P, \mathrm{I}, 33$. 
requisito más para que se alcanzase esa equipolencia de fuerzas: que ambos argumentos contrapuestos no puedan ser falsos a la vez, o como mínimo que no sea posible pensar que ambos son falsos. Es decir, sólo habrá suspensión del juicio en virtud del propio PNC y de sus consecuencias, o, para ser más exactos, en virtud de la vigencia del PNC y de la del Principio de Tercio Excluso9.

Hay que añadir también que, como se afirma en el capítulo III del libro IV de la Metafísica, es posible decir que lo mismo se da y no se da en lo mismo al mismo tiempo, pero no es posible creérselo 10 . En efecto, "no es necesario creerse las cosas que uno dice" 11 , pero la creencia en la posición que uno mismo defiende se nos antoja necesaria para considerar a una posición como teoría filosófica y no como una mera argucia sofística. Así, el verdadero negador del PNC debería ser aquél que creyera que A y $\neg$ A se dan al mismo tiempo y en el mismo sentido. Esto, como se ha dicho, es imposible. Pero para el escéptico es doblemente imposible: no sólo lo es por su propia imposibilidad lógico-psicológica, sino también por una cuestión de coherencia. Para quien asume el escepticismo es imposible, no ya creer A y $\neg \mathrm{A}$, sino simplemente creer A o creer $\neg \mathrm{A}$. El escéptico no puede ser un honesto negador del PNC, como nadie puede serlo, pero además si negase el principio sin honestidad tendría que dejar de ser escéptico. Por tanto, por un lado, necesita el principio para su argumentación basada en la contraposición de argumentos, y por otro, esa contraposición en la que sustenta sus argumentos no conduce a creer A y $\neg \mathrm{A}$, sino que, por el contrario, lleva a no poder afirmar siquiera $\mathrm{A}$ o $\neg \mathrm{A}$, a no poder creer ni A ni $\neg \mathrm{A}$, esto es, a suspender el juicio.

Con todo, la similitud de posturas entre el oponente aristotélico de Metafisica IV y el escepticismo tardío no es inexistente. Excluyendo el hecho de que los escépticos no niegan el principio, la mecánica interna que aparece reflejada en los textos bien podría corresponder a la de las argumentaciones de cualquiera de los seguidores de Enesidemo. Parece, pues, imprescindible atribuir el origen de esa coincidencia a algo más que la mera casualidad, y por ello los esfuerzos de una gran parte de los intérpretes del pasaje se han orientado a la comparación de los argumentos y posiciones del oponente de Aristóteles con los de aquellos filósofos y escuelas que de un modo $\mathrm{u}$ otro pudieron influir en la conformación expositiva del pensamiento escéptico.

Al tratar acerca de la negación del PNC, tanto en el pasaje del libro IV como en el del libro XI, Aristóteles no llega nunca a atribuir inequívocamente la postura cen-

\footnotetext{
${ }^{9}$ De hecho, para que el escéptico no viole el Principio de Tercio Excluso en su argumentación es necesario introducir la equipolencia. El razonamiento sería, pues, el siguiente: aunque la cosa sea A o noA (Principio de Tercio Excluso), yo no creo A ni creo no-A porque las evidencias a favor de ambas afirmaciones tienen la misma fuerza persuasiva.

101005 b $20-30$.

111005 b 25.
} 
tral contra la que argumenta a un filósofo o escuela concretos. Esto no significa que se abstenga por completo de mencionar nombres propios. Encontramos, por ejemplo, vinculado al tema de la contradicción, el nombre de Heráclito ${ }^{12}$, y el de Anaxágoras ${ }^{13}$, entre otros. Cualquiera de ellos encaja a la perfección con la referencia a "los filósofos de la naturaleza" que aparece en 1006 a 2; sin embargo, esta mención por parte de Aristóteles no supone que únicamente quienes practicaron este tipo de filosofía puedan ser considerados como negadores del PNC. El principio mismo no se aplica exclusivamente a la física, sino que tiene implicaciones metafísicas, lógicas, ontológicas y epistemológicas.

Indirectamente, las proyecciones epistemológicas del principio adquieren una gran importancia en Aristóteles debido a que, como decíamos, el principal tipo de negación contra el que combate no será la del filósofo físico, que ejemplificarían Heráclito y Anaxágoras, sino la negación de la contradicción aplicada sobre el conocimiento humano. Esto ha llevado a sopesar la posibilidad de que fuera otro tipo de filósofo el que mejor encarnase la postura del interlocutor de Aristóteles. Se ha propuesto ${ }^{14}$, por ejemplo, a los integrantes de la escuela de Megara, en base a las posiciones atribuidas a ellos por Simplicio, a las referencias al afán dialéctico ${ }^{15}$, y la mención de la ciudad de Megara en 1008 b 14-1516. También el propio Pirrón ha aparecido vinculado al pasaje aristotélico, hipótesis que hemos discutido en otro lugar ${ }^{17}$. Y por supuesto, la posición relativista ha sido uno de los candidatos más habituales propuestos por los especialistas en el tema.

Sobre esta última posibilidad, resulta un indicio importante a su favor el contexto en el que aparece la negación del principio. En efecto, tras la refutación de los negadores del PNC, y vinculado con ella, lo que se expone a continuación en el libro IV de la Metafisica es la refutación de las posiciones relativistas 18 .

Además, la mutua implicación del relativismo en sus manifestaciones más extremas y la negación del PNC es difícil de cuestionar. Es un hecho que hay opi-

\footnotetext{
121005 b 24-5; 1062 a $30-1062$ b 7; 1063 b 25.

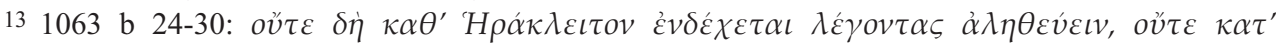

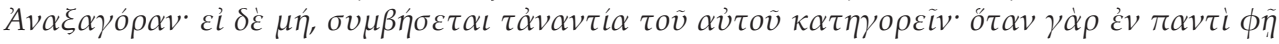

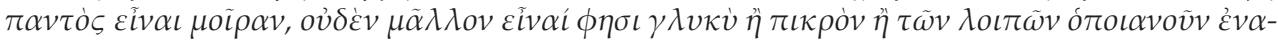

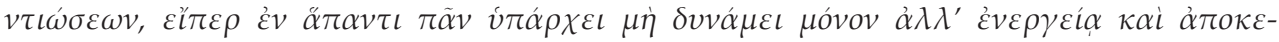
$\kappa \rho \iota \mu ́ v o v$.

${ }^{14}$ Maier, H. Die Syllogistik des Aristoteles. (2 vol.) Tübingen, 1900: ii, pt. 2, 6 y ss.

15 Cfr. 1009 a 20-21, 1011 a 4 y 1011 b 2-3. Los megáricos, sobre todo a partir de Eubulides, eran conocidos particularmente como amantes de la controversia y de la erística.

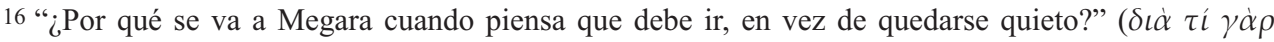

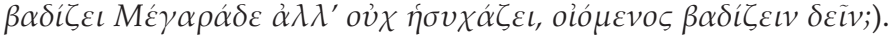

17 Véase Pajón Leyra, I. Categorías y supuestos del escepticismo pirrónico. [Edición digital] Universidad Complutense de Madrid, Servicio de publicaciones, 2011: 275-293. $<$ http://eprints.ucm.es/12660/>.

181009 a $5-1011$ b 23.
} 
niones contrarias e incluso contradictorias entre sí. Si todas las opiniones son, por igual, verdaderas, habrá de admitirse que tanto quien opine A como quien opine $\neg \mathrm{A}$ estarán por igual en la verdad 19 . Por tanto, el relativismo parece requerir algún tipo de supresión o derogación del principio de no contradicción. Ese es el motivo por el que Aristóteles afirma que "de esta misma opinión" -la negación del principio"deriva también la doctrina de Protágoras" 20.

Ha de tenerse en cuenta que el discurso sofístico es ante todo un instrumento de persuasión. Su fin es la convicción, la fascinación e incluso el engaño. Su ámbito de aplicación, eminentemente dialéctico, es un juego de subjetividades hablanteoyente u orador-auditorio en el que la verdad imparcial y objetiva no tiene lugar. En el lógos sofístico no hay búsqueda de adecuación con las cosas, ya que la relación significante-significado ha perdido importancia frente a los efectos puramente prácticos del discurso. La afirmación de que todas las opiniones son verdaderas tiene sentido en este contexto. Sin referente de contrastación, toda opinión es igualmente injustificada. La elección entre ellas no podrá, pues, basarse en criterios valorativos objetivos. Por lo que se impone la necesidad de buscar otra forma de elección.

En sus Antilogías, Protágoras desarrollaba la idea de que cualquier asunto es susceptible de una doble argumentación de signo opuesto. La decantación por uno u otro signo, eliminado el referente veritativo, es indiferente de por sí. El papel del sofista no será, pues, la contrastación, sino el desarrollo de las técnicas dialécticas y retóricas necesarias para forzar la decantación del auditorio por uno u otro argumento con fines prácticos.

De este modo, la refutación de cualquier intento de negar el PNC respondería a la necesidad de arrebatar un posible argumento a un rival dialéctico real. Las filosofías de Heráclito y Anaxágoras las trae a colación Aristóteles por ser los que intro-

19 Vid. 1009 a 12-16: “y si esto es así, las cosas que parecen serán todas verdaderas (los que están en la verdad y los que están en el error mantienen, desde luego, opiniones contrarias entre sí; por tanto, si las cosas son de este modo, todos están en la verdad). Es claro, pues, que ambas doctrinas derivan

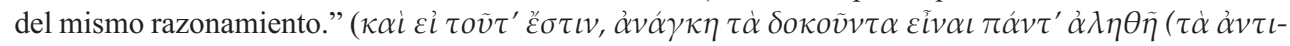

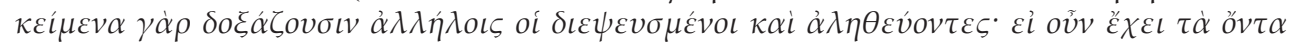

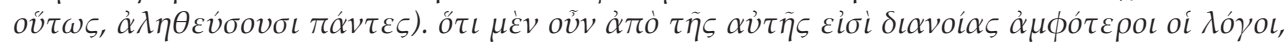
$\left.\delta \tilde{\eta} \lambda o v^{*}\right)$

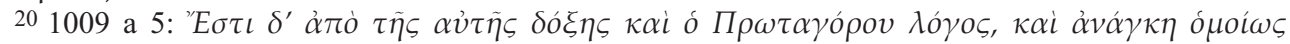

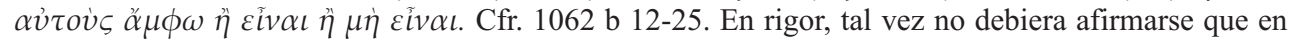
la doctrina de Protágoras se incumple el principio de no-contradicción, puesto que carece de un concepto de verdad absoluta. Su ánthropon metrón pánton (DK 80 B 1) implica que todas las opiniones son verdaderas, pero no respecto de lo mismo. Cada opinión es verdadera para quien la sostiene en tanto que la sostiene, pero no por ello la opinión sostenida por un hombre ha de ser verdadera para otro. Así, el relativismo puede entenderse más como un ataque contra la noción tradicional de verdad que contra la de contradicción, aunque la de contradicción caiga en gran medida al desaparecer la de verdad. Esto, sin embargo, no es óbice para que su ataque contra la noción de verdad no pueda considerarse como constreñido al ámbito de la epistemología, ya que el fenomenismo relativista es plenamente ontológico. 
dujeron en el pensamiento griego las bases sobre las que pudo asentarse y construirse el relativismo fenomenista, pero el objetivo fundamental y último de su crítica, ese que en todo momento transmite la sensación de ser una fuerza viva vigente dentro de la polémica filosófica contemporánea del propio Aritóteles, no puede ser otro que el relativismo.

Por lo que respecta al escepticismo posterior a Enesidemo, su postura ante el PNC será en cierta medida ambigua. En sentido explícito, el escéptico no puede admitir principios ontológicos. Su filosofía siempre es expuesta de modo que quede claro que no consiste en la exposición y defensa de una doctrina ${ }^{21}$, y la admisión del PNC podría considerarse como el punto a partir del cual construir y asentar un cuerpo doctrinal equivalente al de cualquier escuela dogmática. Así pues, no hay en el escepticismo pirrónico ninguna pretensión de aceptar el PNC. Pero sin embargo su derogación también constituye una forma de afirmación de una tesis plenamente cargada de consecuencias ontológicas. Podría considerarse que la respuesta natural del escéptico ante este dilema ha de ser suspender el juicio respecto de la validez o no validez del PNC. De este modo, ninguna consecuencia ontológica podría extraerse de la postura escéptica. Pero una posición semejante, pese a contar con la ventaja de conservar una aparente neutralidad ontológica, no encuentra más que dificultades:

1) En primer lugar, caería en el argumento aristotélico expuesto en 1006 a 21 1007 a 21. Cualquier afirmación unívoca implica ya un compromiso con el PNC. Pero este es el menor de los problemas que presenta.

2) Además, una afirmación semejante, como ya se ha mostrado, es incapaz de conducir al escepticismo. Si no se afirma que es imposible pensar que lo mismo se da y no se da en lo mismo22, entonces tampoco será posible establecer una contraposición entre apariencias enfrentadas que impida la elección por una de ellas respecto del mismo sujeto, puesto que podrá optarse por las dos -esto es, por la afirmación simultánea de los contrarios- de tal modo que ningún argumento escéptico podría conducir a la ataraxía a través de la epoché.

3) La dimensión significativa de cualquier afirmación se disolvería, con lo que perdería su sentido la comunicación. El escéptico se vería imposibilitado para argumentar contra el dogmático. La fuerza persuasiva de cualquier intento de argumentación sería nula, y toda pretensión escéptica de hacer proselitismo de su propia posición resultaría vana. Incluso toda enseñanza del modo de filosofar escéptico carecería de sentido.

${ }^{21} H P$, I, 13-18.

22 Es decir, si no se afirma la vigencia al menos de la dimensión psicológica del PNC. 
Así pues, el problema no puede resolverse recurriendo a la simple suspensión del juicio en lo referente a la validez del PNC. El escéptico necesita de ese principio aunque no pueda afirmarlo. Y lo necesita porque la noción misma de contradicción subyace a la asunción del escepticismo.

Sólo admitiendo que la realidad se muestra ante nosotros siempre de una manera contradictoria y, al tiempo, que no puede ser de hecho contradictoria (o al menos que no puede asumirse esa contradictoriedad), el escepticismo será posible. En la formulación de la teoría filosófica escéptica que Sexto nos ha transmitido, el uso de la contradicción es constante. La contradictoriedad aparece casi en cada argumento en defensa de la postura escéptica. Pero no es una contradictoriedad afirmada respecto de la realidad, lo que supondría contravenir la intención escéptica de no hablar de nada más allá de lo que al propio escéptico le aparece, esto es, el fenómeno. Se trata de una contradictoriedad expuesta como característica intrínseca de la apariencia misma 23 . De la realidad, en cambio, nada se afirma ni se niega, con lo que parece quedar salvaguardada la neutralidad ontológica de la posición pirrónica.

Pero desde esa neutralidad la caracterización de la apariencia como contradictoria carecería de significado. Se requiere, además, de la asunción de la no-contradicción como requisito imprescindible de la realidad, cualquiera que ésta sea.

Sexto, como cualquier otro escéptico pirrónico, ha de transgredir su pretendida neutralidad respecto del modo de ser de las cosas y suponer la necesidad de algún tipo de no-contradicción. Sólo admitiéndolo y respetándolo como "regla del pensamiento" y "criterio de significación del discurso" podrá dar validez a sus propias estrategias argumentativas.

La forma más clásica del argumento escéptico sería, quizá, la siguiente:

A aparece $\mathrm{p}$ en un momento $\mathrm{t}$

A aparece $\neg p$ en un momento $t$ '

$\overline{\text { No es posible decidir entre } \mathrm{p} \mathrm{y}} \neg \mathrm{p}$ respecto de A

En este argumento encontramos que la contradicción está circunscrita al ámbito fenoménico, ya que "p" y " $\neg$ p" son propiedades de la apariencia de A. Y ni siquiera en la propia apariencia incumplen el PNC, puesto que se sitúan en dos momentos distintos ( $\mathrm{t}$ y t'). Pero para que el argumento funcione ha de añadirse también alguna premisa oculta nunca explicitada por el propio Sexto, ya que de lo contrario de las premisas no podría inferirse la conclusión. Habría de añadirse, en primer lugar, una premisa no explicitada en esta ocasión pero sí consciente y tema-

\footnotetext{
${ }^{23}$ Esta forma de asunción de la contradicción, limitada a la apariencia y al fenómeno individual y subjetivo, parece, como veremos, más próxima a la dimensión psicológica del PNC que a la ontológica.
} 
tizada a lo largo de la filosofía escéptica: la de la equipolencia de fuerzas entre la argumentación a favor de $\mathrm{p}$ y la argumentación a favor de $\neg$ p.

Pero habrá de añadirse, además, una cuarta premisa que introduzca el carácter de incompatibilidad entre $\mathrm{p} \mathrm{y} \neg$ p, premisa que estará formulada en base a la asunción de alguna de las versiones del PNC. Nuestro primer impulso será suponer que esta última premisa oculta es la versión ontológica fuerte del principio. De ser así, el argumento escéptico quedaría formulado como sigue:

A aparece $p$ en un momento $t$

A aparece $\neg p$ en un momento $t$ '

Hay equipolencia entre $\mathrm{p}$ y $\neg \mathrm{p}$

No es posible que A sea $\mathrm{p} \wedge \neg \mathrm{p}$

No es posible decidir entre $\mathrm{p} \mathrm{y} \neg \mathrm{p}$ respecto de A

De admitirse que fuese esta la premisa oculta del razonamiento supondría, además, la admisión de las siguientes consecuencias difícilmente compatibles con el escepticismo:

1) La vigencia del PNC en el ámbito de la realidad.

2) La imposibilidad de un tercer valor de verdad, ya que A sólo puede ser $\mathrm{p} o$ $\neg$ p, esto es, la propiedad "p" sólo puede ser V o F respecto de A. Un tercer valor de verdad que permitiese que A no fuese ni p ni $\neg$ p anularía el argumento al sumir la realidad en la indeterminación.

3) Como consecuencia de lo anterior, el escéptico concebiría la realidad de una manera determinada (aunque desconocida). En este punto la filosofía pirrónica se alejaría de Pirrón y de su concepción indeterminista de la realidad, hacia una visión de la indeterminación epistemológica y no ontológica.

4) También como consecuencia del punto segundo, habría de admitirse la necesidad de la verdad. No sólo estamos obligados a considerar $\mathrm{p}$ o $\neg$ p respecto de $\mathrm{A}$, sino que tenemos que considerar que necesariamente una de las dos opciones será verdadera. Pero también que necesariamente su contraria será falsa 24 .

\footnotetext{
${ }^{24}$ Cfr. Calvo, T. «El principio de no-contradicción en Aristóteles», Méthexis I, 1988: 54: "dos predicaciones opuestas (...) no pueden ser a la vez verdaderas del mismo sujeto. Esto quiere decir que si una de ellas es verdadera la otra es necesariamente falsa y, por tanto, que el conjunto de todos los enunciados posibles acerca de las cosas incluye necesariamente enunciados que son falsos. La tesis aristotélica de la no-contradicción comporta, pues, la tesis de que hay, o mejor, tiene que haber enunciados que son verdaderos y enunciados que son falsos o, de otro modo, que no todos los enunciados son verdaderos aunque algunos tienen que serlo". Nótese que esa consecuencia del PNC es la contradicción directa de la tesis fundamental de todo relativismo - esto es, que todos los enunciados tienen el mismo
} 
Además, si, teniendo en cuenta las distintas formulaciones del PNC, nos preguntamos cuál sería la formulación concreta que el escéptico habría admitido tácitamente, nos podríamos inclinar por la que establece o afirma algo no acerca de las cosas, sino acerca del discurso sobre las cosas. El escéptico no pretendería hacer una aserción del tipo de aquella con la que Aristóteles establece el PNC desde el punto de vista de la realidad en 1005 b 19-20 ("es, pues, imposible que lo mismo se dé y no se dé en lo mismo a la vez y en el mismo sentido"25), pues de hacerlo caería en una violación demasiado evidente de su pretensión de mantener la neutralidad en todo aquello que rebase la esfera de lo que a él le aparece. La formulación del PNC en este sentido es "demasiado ontológica" para el escéptico; refiere con excesiva claridad hacia el ser de las cosas.

Sin embargo, cabe aún otra alternativa pocas veces contemplada a este respecto: Para la imposibilidad de decisión entre $\mathrm{p}$ y $\neg \mathrm{p}$ no es imprescindible la afirmación ontológica del principio $\neg(\mathrm{p} \wedge \neg \mathrm{p})$, ni siquiera en su aspecto predicativo, sino que bastaría con que se admitiera que no es posible pensar $p \wedge \neg p$; esto es, bastaría con admitir la formulación psicológica del PNC, quedando el argumento expuesto como sigue:

A aparece $\mathrm{p}$ en un momento $\mathrm{t}$

A aparece $\neg p$ en un momento $t$ '

Hay equipolencia entre $\mathrm{p}$ y $\neg \mathrm{p}$

No es posible pensar que A sea $\mathrm{p} \wedge \neg \mathrm{p}$

No es posible decidir entre $\mathrm{p} \mathrm{y} \neg \mathrm{p}$ respecto de A

Para el escéptico pirrónico esta formulación del argumento resultaría con mucho menos incómoda que la anterior, ya que la dimensión psicológica, en lugar de hacer referencia al modo en que las cosas mismas son por naturaleza, remite al modo en que éstas se presentan psicológicamente a un sujeto, esto es, el modo en que las cosas le aparecen.

El argumento escéptico, de este modo, no rebasaría el ámbito de lo fenoménico para interferir en el de lo natural, sino que se limitaría a la mera apariencia. Sin embargo, para que esto sea así es necesario concebir la formulación psicológica del PNC de un modo que no sea aquel en el que Aristóteles lo concibe, esto es, el de corolario del principio ontológico, pues de lo contrario la afirmación del hecho de que "no es posible pensar que A sea $\mathrm{p} \wedge \neg \mathrm{p}$ " conducirá de inmediato a la de que "no es posible que A sea de hecho $\mathrm{p} \wedge \neg \mathrm{p}$ ", ya que, admitida la relación de dependencia

valor de verdad- de modo que cualquier posición filosófica que asuma ese principio -incluido el escepticismo- será por necesidad "antirrelativista".

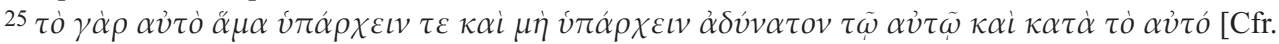
Calvo, T. Op. cit.: 53] 
entre ambas formulaciones del principio, si no es posible pensarlo es porque no es posible que sea así.

Con independencia de esta posible vinculación, el hecho de que el escéptico incluya en su razonamiento esta dimensión del PNC tiene también sus propias consecuencias, que consideramos que son las que es legítimo asumir como irremediablemente unidas a todo argumento escéptico:

1) Vigencia del principio de no-contradicción en su vertiente psicológica en todo el ámbito de lo fenoménico. Esta forma del principio, así, actuaría como ley universal del páthos, no en el mero sentido de apariencia (puesto que las cosas aparecen como de hecho contradictorias) sino en el de parecer propio de todo sujeto.

2) Esta asunción del PNC reducida al ámbito de lo fenoménico parece llevar aparejada una aceptación del principio de tercio excluso también limitada a ese ámbito, esto es, la aceptación de que es necesario creer $\mathrm{p} \vee \neg \mathrm{p}$ para optar racionalmente.

3) También aparece implicada en esta formulación del argumento la no necesidad de aceptación de la existencia de "verdades lógicas", "ontológicas" o "epistemológicas" en sentido tradicional.

4) Como contrapartida al punto anterior, al escéptico le surge la necesidad de delimitar un sentido "fenoménico" o "apariencial" de la verdad que dé sentido a los argumentos basados en su propio páthos.

5) Por otra parte, la aceptación del punto 2 hace recaer en exclusiva la decisión entre $\mathrm{p}$ o $\neg \mathrm{p}$ sobre cuál de las dos alternativas sea creída, en lugar de sobre criterio de verdad alguno. Por tanto, el argumento escéptico formulado de este modo será eminentemente dialéctico, y su éxito o fracaso dependerá del papel de la persuasión.

6) Por último, asumiendo el PNC sólo en su dimensión psicológica el escéptico puede afirmar que "le aparece", y que por tanto no afirma nada más allá de su apariencia. Pero para que su razonamiento funcione no basta con su afección individual, sino que tendría que postular una ley universal válida para todas las afecciones: "Nadie puede pensar $\mathrm{p} \wedge \neg p$ ". Y esta afirmación sí que supone ir más allá de los límites de la propia afección.

Esta "ley universal" de la apariencia es, en cierto modo, una afirmación sobre la naturaleza; en concreto, sobre el modo en que por naturaleza nos aparecen las cosas. Sin su admisión, el argumento escéptico carecería de validez, ya que el interlocutor podría afirmar que cree de veras simultáneamente $\mathrm{p} \mathrm{y} \neg$ p. Pero si el escéptico la admite transgrede con mucho su pretensión de neutralidad sobre cosas no manifiestas. En primer lugar, la máxima de que "nadie puede pensar $\mathrm{p} \wedge \neg p$ " supone 
que los fenómenos subjetivos que se dan a los distintos sujetos se nos dan según una estructura común e invariable, válida para cualquier sujeto.

Esta estructura común no se limitaría a la mera incompatibilidad simultánea entre la creencia de p y la creencia de $\neg$ p. Además supondría que la no creencia en $\neg p$ es condición de posibilidad de la creencia en $p$ (esto es, que es necesario no creer que algo es falso para creer que es verdadero), y que esta condición de posibilidad es necesaria pero no suficiente (esto es, que puede no darse la creencia en p y tampoco la creencia en $\neg$, o dicho de otro modo, que es posible la suspensión del juicio).

En efecto, esta concepción de la estructura de los pareceres subjetivos es imprescincible para que el argumento escéptico tenga sentido, puesto que de lo contrario sería imposible la epoché. La postura escéptica será precisamente no creer $\mathrm{p}$ y tampoco creer $\neg$ p. Y esto es posible porque aunque el PNC en su formulación clásica $\neg(\mathrm{p} \wedge \neg \mathrm{p})$ supone que $\mathrm{p} \vee \neg \mathrm{p}$, en su dimensión sólo psicológica el hecho de que nadie pueda creer a la vez $p$ y $\neg$ p no implica que no pueda no creer ni p ni $\neg$ p. Esto es, al "principio psicológico de no-contradicción" no está vinculada una suerte de "principio psicológico de tercio excluso".

\section{Ignacio Pajón Leyra}

Departamento de Historia de la Filosofía

Universidad Complutense de Madrid

ipajon@filos.ucm.es 